\title{
Systematic search for Wolf-Rayet stars in young starbursts
}

\author{
Orly Kovo \\ School of Physics $\mathcal{B}$ Astronomy, Tel Aviv University, Israel \\ Thierry Contini \\ European Southern Observatory, Garching, Germany
}

\begin{abstract}
Preliminary results from a systematic search for WR stars in starburst galaxies are presented. We report new detections of WR spectral features (the 'WR bump' around $\sim 4700 \AA$ ) in eight objects.
\end{abstract}

\section{Introduction}

Wolf-Rayet galaxies (see review by P.S. Conti, these Proceedings) are the ideal laboratories to derive stellar populations and burst properties in nearby starforming regions (e.g., Schaerer, Contini \& Kunth 1999). Since the initial catalogue listing 37 galaxies (Conti 1991), the number of known WR galaxies has grown rapidly to about 130 in the recent compilation of Schaerer, Contini \& Pindao (1999). Most of them have been discovered serendipitously and only two systematic searches have been performed so far with limited success (Kunth \& Joubert 1985; Masegosa et al. 1991). Due to the high sensitivity of CCDs mounted on 2-meter class telescopes, a systematic search for WR galaxies can now be undertaken.

\section{Results}

From the samples of $\mathrm{H}$ II galaxies (Terlevich et al. 1991) and starburst nucleus galaxies (Contini et al. 1998), we selected galaxies with an age of the burst in the range between 3 and $6 \mathrm{Myr}$, which corresponds to the predicted WR phase in star-forming regions (Meynet 1995; Schaerer \& Vacca 1998). Long-slit spectra of these galaxies were obtained in two runs (March 1996, August 1997) at the ESO $1.52 \mathrm{~m}$ telescope. The data were acquired with the $\mathrm{B} \& \mathrm{C}$ spectrograph, giving a spectral coverage of $\sim 3000-9000 \AA$ with a resolution of $\sim 10 \AA$. The slit was oriented in order to cover $\mathrm{H}$ II regions with known young starbursts. The total integration time for each galaxy ranges from $\sim 45$ to 120 min. A preliminary list of galaxies with spectral features around $4700 \AA$ is given in Table 1 .

The most obvious signature of WR stars in the integrated spectrum of extragalactic H II regions is the broad He II $\lambda 4686$ emission line. This line is clearly detected in eight star-forming regions distributed in six galaxies of our sample. The fainter broad C IV $\lambda 5808$ line, mainly produced by WR stars of WC subtype, is suspected in the spectrum of Fairall 44. A narrow, nebular in 
origin, He II $\lambda 4686$ line is observed in 16 starburst regions, including most of the new WR galaxies. The origin of the nebular He II $\lambda 4686$ emission in starburst galaxies is not yet clearly understood, since its excitation requires a relatively hard ionizing spectrum, but it could be intimately linked with the appearence of hot WR stars (Schaerer 1996).

To test this hypothesis, it is crucial to measure the relative contributions of the broad WR and narrow nebular emission in the He II $\lambda 4686$ line, and to search for weak WR emission features (e.g., C IV $\lambda 5808$ for WC stars) in those galaxies for which nebular He II $\lambda 4686$ emission has been detected but WR signatures have not been seen. This is one of the purposes of our ongoing project (Kovo \& Contini, in preparation).

Table 1. Starburst regions with broad and/or nebular He II $\lambda 4686$.

\begin{tabular}{lrrrrcc}
\hline galaxy & & $\begin{array}{r}\text { R.A. } \\
(1950)\end{array}$ & $\begin{array}{r}\text { Dec. } \\
(1950)\end{array}$ & $\begin{array}{r}v_{\text {rad }} \\
\left(\mathrm{km} \mathrm{s}^{-1}\right)\end{array}$ & $\begin{array}{r}\text { He II } \lambda 4686 \\
\text { broad } \\
\text { nebular }\end{array}$ \\
\hline Mrk 960 & A & 004835 & -124300 & 6411 & & + \\
& B & & & & + & + \\
ESO 566-8 & A & 094239 & -192854 & 9750 & & + \\
Mrk 712 & A & 095359 & +155234 & 4550 & + & + \\
UM 462 & A & 115004 & -021128 & 986 & & + \\
& B & & & & & + \\
UM 469 & B & 115439 & +024510 & 17388 & & + \\
Mrk 1318 & A & 121636 & +040807 & 1527 & & + \\
UM 533 & A & 125724 & +021911 & 874 & & + \\
Tol 30 & A & 130303 & -280912 & 2099 & + & + \\
Tol 1400-411 & A & 140018 & -410811 & 513 & + & + \\
& B & & & & + & + \\
ESO 513-11 & A & 145731 & -261506 & 5115 & + & + \\
& B & & & & + & + \\
Fairall 44 & A & 180919 & -574448 & 4948 & + & + \\
Tol 1924-416 & A & 192429 & -414036 & 2837 & & + \\
\hline
\end{tabular}

\section{References}

Conti, P.S., 1991, ApJ 377, 115

Contini, T., Considère, S., Davoust E. 1998, A\&AS 130, 285

Kunth, D., Joubert, M. 1985, A\&A 142, 411

Masegosa, J., Moles, M., Del Olmo, A. 1991, A\&A 244, 273

Meynet, G. 1995, A\&A 298, 767

Schaerer, D. 1996, ApJ 467, L17

Schaerer, D., Contini, T., Kunth, D. 1999, A\&A 341, 399

Schaerer, D., Contini, T., Pindao, M. 1999, A\&AS 136, 35

Schaerer, D., Vacca, W.D. 1998, ApJ 497, 618

Terlevich, R., Melnick, J., Masegosa, J., Moles, M., Copetti, M.V.F. 1991, A\&AS 91, 285 